\title{
Espace et identité culturelle dans la réception de la téléréalité en Suisse romande
}

\section{Charlotte Bouchez}

\section{(2) OpenEdition \\ 1 Journals}

Édition électronique

URL : http://journals.openedition.org/edl/498

DOI : $10.4000 /$ edl. 498

ISSN : 2296-5084

Éditeur

Université de Lausanne

\section{Édition imprimée}

Date de publication : 15 mai 2013

Pagination : 205-222

ISBN : 978-2-940331-32-1

ISSN : 0014-2026

Référence électronique

Charlotte Bouchez, « Espace et identité culturelle dans la réception de la téléréalité en Suisse

romande ", Études de lettres [En ligne], 1-2 | 2013, mis en ligne le 15 mai 2016, consulté le 19 décembre 2020. URL : http://journals.openedition.org/edl/498 ; DOI : https://doi.org/10.4000/edl.498 


\section{ESPACE ET IDENTITÉ CULTURELLE DANS LA RÉCEPTION DE LA TÉLÉRÉALITÉ EN SUISSE ROMANDE}

Cet article expose un aspect particulier de la réception de la téléréalité en Suisse romande qui a trait au rôle des discours de presse dans la construction d'un imaginaire culturel localement déterminé. En proposant des éléments descriptifs et normatifs à propos des premiers programmes français et suisses diffusés dans la région romande, ils participent à cette territorialisation de l'identité culturelle. Plus particulièrement, nous étudions la façon dont la Télévision Suisse Romande (TSR) se positionne comme chaîne publique romande en radicalisant sa distinction avec les chaînes commerciales françaises, la téléréalité fonctionnant comme support de cette revendication.

Dans le cadre de la recherche de doctorat que nous menons sur la réception de la téléréalité en Suisse romande, nous visons à dégager les mécanismes qui permettent qu'une telle catégorie générique soit reconnue et articulée dans l'espace social, en particulier par le biais des discours portant sur une telle production, et à montrer comment un tel objet médiatique exemplifie les mécanismes sous-jacents à la mondialisation culturelle.

Cette intégration de l'ensemble des concepts, des valeurs morales et des connaissances associés à la téléréalité dans l'espace social s'opère par la combinaison de plusieurs médias (presse, radio, télévision), qu’ils soient envisagés comme des supports de diffusion des programmes ou comme des vecteurs de discours portés sur la téléréalité. Nous avons choisi d'étudier en particulier la presse d'information qui, en tant que lieu de publication de discours légitimés, diffuse des cadres conceptuels, mais aussi des normes d'évaluation et des indications de lecture qui participent à déterminer la façon dont les spectateurs appréhendent un tel objet télévisuel. Il ne s’agit cependant pas de considérer le champ social 
romand comme un lieu de circulation d'opinions homogènes, mais comme un espace de positionnements pour les individus et les institutions. Ces positionnements se concrétisent en partie en recourant à des énoncés, potentiellement contradictoires, associés à des objets perçus comme communs (en l'occurrence, la téléréalité) qui circulent et sont mobilisés dans les situations discursives qui structurent la socialité ${ }^{1}$.

La réception de la téléréalité en Suisse romande est ainsi l'occasion pour les principaux acteurs institutionnels de ce dispositif - en particulier, les journalistes et les représentants de la Télévision Suisse Romande (TSR) - de définir leur appartenance à l'espace culturel romand dont ils contribuent également à produire une certaine définition. Profitant de l'opportunité offerte par la thématique du colloque dans lequel s'est originellement inscrite cette contribution, le présent article rend compte des processus de spatialisation qui interviennent dans la construction et l'expression de l'identité culturelle romande au sein des discours consacrés à la téléréalité dans la presse généraliste romande ainsi que dans certains programmes de téléréalité produits par la TSR ${ }^{2}$.

\section{Téléréalité et mondialisation: entre standardisation et ancrage local}

Jusqu'au milieu des années 80 , le modèle qui prévaut dans la plupart des pays est celui d'une télévision nationale, où le territoire couvert par la diffusion (l'espace de diffusion) recouvre à peu près l'espace national,

I. Morley et Robins relèvent ce rôle des médias de masse dans la construction des communautés culturelles: "[i]t has been frequently observed that mass communications have played a fundamental part in the historical development of national cultures and identities. Print and then broadcast media brought into being mass publics who began to imagine the community of the nation and nationalism» (D. Morley, K. Robins, Spaces of Identity, p. 174 sq.).

2. L'analyse porte sur les émissions suivantes: Mayen 1903 (2003), Super Seniors (2005), Dîner à la ferme (2009 et 2010) et Mon village a du talent (2011). Le corpus est constitué d'articles parus dans les grands quotidiens romands (24 heures, Le Temps, Le Matin, La Tribune de Genève, Le Nouvelliste, L'Express) ainsi que les suppléments de programme TV qui accompagnent les éditions du week-end (TV8 et TVGuide), et deux magazines de société (L'Hebdo et L'Illustré). Les articles ont été sélectionnés grâce au moteur de recherche et d'indexation Swissdox, en regroupant les sources qui présentaient une occurrence des termes: "real TV», "téléréalité », «Big Brother», "Loft Story» sur une période s'étendant de 1996 à 2008. 
lui-même également déterminé comme un espace homogène au niveau linguistique. On peut ainsi parler d'une télévision calquée sur le mode d'une triade "langue/territoire/culture", dont les chaînes de service public européennes sont emblématiques. Les années 90 sont caractérisées par un ensemble de changements technologiques et législatifs qui, reposant sur une dérégulation du marché de l'audiovisuel, vont mettre en crise ce modèle. Ces mêmes années voient l'émergence de nouveaux produits commerciaux, particulièrement adaptés à cette structure du champ économique lié aux médias: les formats, dont les programmes de téléréalité sont des exemples types ${ }^{3}$. La variabilité d'un même format, qui permet qu'il puisse être vendu internationalement tout en étant adapté aux spécificités du contexte de réception auquel il est destiné, rend ce type de production remarquablement adéquat à cette situation de globalisation médiatique ${ }^{4}$. Certains chercheurs montrent en effet que celle-ci repose sur une tension entre une tendance à l'homogénéisation des pratiques associées aux usages des médias et un mouvement inverse de revendication des différences culturelles et des particularismes régionaux qui sont actifs au sein de l'espace local où s'ancre la communauté ou l'individu ${ }^{5}$.

3. Un format est un modèle, une description d'un programme de télévision. Ce qui est vendu, c'est ce canevas, qui prescrit un certain nombre d'éléments caractérisant l'émission, mais qui peut ainsi être adapté à chaque espace de diffusion où il sera produit (le point principal concerne les participants et le décor, mais la programmation ainsi que la durée des émissions peuvent également varier).

4. Ce modèle est notamment exposé par Appadurai, Après le colonialisme, et Barker, Television, Globalization and Cultural Identities. Dans notre perspective, la mondialisation médiatique est définie d'une part, par un ensemble de processus économiques et légaux qui permettent une circulation accrue des marchandises, des informations et des personnes à l'échelle mondiale et d'autre part, par "l'intensification de la conscience du monde comme un tout» (Ch. Barker, Television, Globalization and Cultural Identities, p. 34).

5. C'est le cas notamment de la revue Médiamorphoses qui a consacré un numéro spécial à Big Brother (septembre 2003), en regroupant les analyses comparatives menées sur plusieurs versions de ce format. On peut aussi relever les propos d'Elodie Kredens: "En ce qui concerne la télé-réalité, s’il existe bien une standardisation des "contenants", autrement dit de ses concepts, il semble bien exagéré de conclure définitivement à une homogénéisation des "contenus". [...] Il existe une dynamique commune qui permet à l'émission de s'internationaliser et une variation évidente des conditions de production et des cadres culturels d'interprétation quand bien même le format initial comporte tous les attributs des industries culturelles.» ("La télé-réalité entre adaptabilité des formats et spécificités nationales", p. 1). 
La plasticité des programmes de téléréalité et les particularités de leur réception dans la presse et dans l'espace social en font des objets particulièrement révélateurs pour étudier les conséquences de la mondialisation sur les communautés culturelles.

Explorant cette problématique pour le cas suisse, notre recherche permet de montrer l'effectivité de cette tension dans la réception des programmes de téléréalité, qui vont être l'enjeu de processus de revendication de particularismes identitaires localisés et territorialisés.

\section{Situation frontalière et particularisme identitaire en Suisse romande}

La Suisse, bien que ne présentant pas d'homogénéité linguistique au niveau national, repose sur un modèle analogue à celui qui vaut pour la majorité des chaînes publiques européennes à l'échelle de ses régions linguistiques. Chacune d'entre elles est couverte par une chaîne publique qui occupe une place dominante en termes de marché dans sa région linguistique. Au niveau de la réception des productions culturelles, que cela concerne la presse, le cinéma ou la télévision, chaque région linguistique est davantage en contact avec les productions des pays frontaliers (en particulier dans le cas du Tessin et de la région romande) qu'avec les productions des autres régions suisses ${ }^{6}$.

L'analyse des premiers articles consacrés aux programmes de téléréalité diffusés dans l'espace européen et en particulier la première émission française, Loft Story, diffusée en 2001 par la chaîne M6, montre comment s'articule cette relation particulière qu'entretiennent les acteurs institutionnels romands avec les chaînes françaises. Ainsi, si le fait que l'offre télévisuelle disponible en Suisse soit principalement composée de chaînes françaises ne semble pas faire question en général, on constate cependant, dans le corpus de réception de Loft Story, la mise en place de stratégies de délimitation d'un espace culturel spécifiquement romand au sein de l'espace global de diffusion de l'émission (qui couvre la région formée de la France, la Suisse romande et la Belgique francophone). La réception du programme français est l'occasion de délimiter, au sein

6. Voir à ce propos: M. Cucco, "The Borders of the Domestic Market and Their Importance for the Economy of the Film Industry»; M. Cola, "Media Studies and Social Geography». 
de cet ensemble, une région définie selon une limitation qui retrace les contours d'un espace identitaire caractérisé en termes de culture et de tradition télévisuelle singulières, différent en cela de la France. Dans ces discours, la Suisse est représentée comme un espace culturel menacé, dans sa spécificité, par les productions de son voisin. La perméabilité du territoire suisse aux productions culturelles exogènes se traduit dans ces discours par le recours au motif lexical de l'invasion, fréquemment utilisé pour caractériser le lancement de Loft Story: «le succès de Loft Story déborde en Suisse", "raz-de-marée», "déferlante real TV». L'espace romand est construit comme ce qui peut être envahi, dont la frontière, non-définie si ce n'est par le fait d'être le lieu où s'ancre ce point de vue sur une production perçue comme invasive et anxiogène, est en voie d'être franchie par ces programmes:

Loft Story constitue la première version française de cette téléréalité qui investit depuis deux ans les écrans du monde entier. M6 est la première grande chaîne francophone à oser se lancer dans la «télé trou de serrure», ce genre qui fait de nous des voyeurs ${ }^{7}$.

Aujourd'hui, le concept fait son apparition en France, sur M6, avec Loft Story. Nous avons tous considéré Big Brother d'un petit air légèrement supérieur. Facile, les images n'étaient pas disponibles dans notre langue et dans nos lucarnes. Pas moyen d'être voyeurs sur ce coup-là. Quel sera notre comportement ce soir face aux images de M6 ? ${ }^{8}$

Ancrant leur point de vue dans le même espace local que les potentiels spectateurs auxquels ils s'adressent, et connotant cet ancrage sur un mode défensif, les journalistes spécifient ainsi une identité culturelle commune avec leur lectorat, susceptible de favoriser une réception similaire de l'émission par le partage de valeurs et de savoirs communs.

7. M. Imhof, "Non à la télé "trou de serrure" et aux "émissions poubelles" ", p. 39.

8. P. Schouwey, «Mon œil», p. 4. 


\section{La TSR et la notion d'identité de chaîne romande}

Face à l'«altérité menaçante " ${ }^{9}$ que représente la téléréalité française, la TSR est construite comme une instance susceptible de manifester (voire d'incarner) cette position identitaire.

La conception que se font les représentants de la chaîne de leur mission de service public est exposée dans nos sources au sein d'un ensemble conceptuel qui met en relation le service public et le concept d'identité. Celui-ci présente deux acceptions complémentaires: il désigne l'ensemble des éléments qui caractérisent la chaîne et permettent sa distinction et sa reconnaissance parmi les autres entreprises médiatiques (en particulier les chaînes françaises). Les modalités énonciatives des articles participent de cette logique en construisant la TSR comme une entité déterminée et unifiée, dotée d'une quasi personnalité incarnée dans la figure de son directeur, ou, pour le cas particulier des articles consacrés aux émissions de la TSR, dans celle de leur productrice, Béatrice Barton ${ }^{10}$. La notion d'identité désigne également la relation de similitude de la chaîne avec les (ses) téléspectateurs. Le discours inaugural du directeur de la chaîne lors de sa prise de fonction est caractéristique de l'agencement de ces deux notions:

Précisément, en conférence de presse, où il a présenté le fruit de son analyse après six mois d'intenses consultations à tous les niveaux de la chaîne, il s'est dit très attaché à cette relation de confiance avec les téléspectateurs romands, une "valeur ajoutée» identitaire sur laquelle la TSR compte s'appuyer dans sa "lutte avec la concurrence des chaînes françaises notamment». Un ancrage romand qui devrait se retrouver

9. Jouant également sur la spatialisation de l'appartenance culturelle, la métaphore guerrière est également très présente dans les articles analysés. Il s'agit ainsi de comparer la région romande à une sorte de fief, de bastion à défendre face à l'invasion. On retrouve des expressions comme "la contre-offensive», "la réplique», "la riposte» ou encore «la TSR prépare ses armes pour contrer la téléréalité».

IO. Au nombre des stratégies communicationnelles qui participent à cette anthropomorphisation de la chaîne, on peut relever l'engagement affectif de la productrice dans la conception de ces programmes, mais aussi dans les relations qu'elle met en place avec les participants, fréquemment mentionné dans les sources. A titre d'exemple, nous relevons le cas d'un article dans lequel l'activité professionnelle est subordonnée au plaisir ressenti: «je pourrai continuer à faire ce que j'aime en ce moment, c'est-à-dire rechercher des nouveaux formats d'émission" (Béatrice Barton, citée dans J.-F. Krähenbühl, "Béatrice Barton, reine de l'information divertissement», p. 4). 
«dans toutes les émissions». Qu'on se rassure: l'identité culturelle, politique, économique et linguistique défendue par Gilles Marchand se veut «moderne, tolérante, ouverte sur le monde, porteuse de projets» ${ }^{11}$.

Via la médiation journalistique, la TSR se présente comme une entreprise dont le statut repose sur la relation qu'elle entretient avec «les téléspectateurs romands». Ainsi, c'est sur la similitude d'appartenance régionale entre l'instance de diffusion et l'instance de réception que semble reposer le succès de la chaîne: le goût du spectateur romand pour un programme romand par rapport à un programme français est fonction du degré de reconnaissance d'un socle culturel commun partagé avec la chaîne. Dans ce cas, la notion d'identité prend le sens d'une relation de similitude, la télévision étant conçue comme le lieu d'un reflet (soit qu'elle reflète le goût et les préoccupations des romands, soit qu'elle constitue, parce qu'elle est l'expression de la même appartenance culturelle, une forme de miroir du mode de vie romand).

Dans une interview parue une semaine après le lancement de Loft Story, le directeur des programmes de l'époque, Raymond Vouillamoz, précise la position de la chaîne en condamnant très clairement l'émission et le genre téléréalité en général pour des questions morales, comme les dangers psychologiques encourus par les participants ou la marchandisation de leur intimité. Reconfigurant l'identité de la chaîne sur le plan éthique, il oppose à la téléréalité une «télévision de proximité, encore plus présente sur le terrain ${ }^{12}$. Dans un second article, il exprime la nécessité de développer "l'information régionale qui échappe à la concurrence française» ou encore «des programmes bien de chez nous ${ }^{13}$. L'appartenance au même espace culturel que les téléspectateurs/ lecteurs se traduit par cet ancrage dans le "chez soi", par le partage d'un point de vue situé dans le territoire identitaire ${ }^{14}$. L'ancrage localisé de

II. N. Richon, "Le nouveau directeur de la TSR opère des changements avec un gant de velours", p. 42.

I2. Raymond Vouillamoz, cité dans J. Elgass, «Le succès de Loft Story déborde en Suisse», p. 33.

13. Raymond Voullamoz, cité dans E. Nicoud, «La TSR résiste à la tempête», p. 34.

I4. Largument de l'origine régionale et de l'identité est d'ailleurs conçu comme une garantie de succès par les représentants de la TSR: «... ce qui marche, ce sont des émissions imaginées en Suisse pour des téléspectateurs suisses" (Raymond Vouillamoz cité dans (ats), "La télé-réalité débarque à la TSR», p. 25). 
l'institution se trouve ainsi articulé avec un ensemble de caractéristiques morales, la "proximité» géographique renvoyant à une similarité de valeurs et d'intérêts postulés comme identiques pour toutes les personnes vivant en Suisse romande, qu'il s'agisse des représentants de la chaîne ou des spectateurs. Cet aspect de la mission de service public qui insiste sur l'ancrage régional est central dans le processus de définition de l'identité de la chaîne romande, dont on verra que les émissions de téléréalité traitées dans cet article présentent toutes un argument procédant de cette conception.

La téléréalité "version TSR»: des programmes emblématiques de la culture romande

Le premier programme de téléréalité produit par la TSR, Le Mayen $1903^{15}$, est l'occasion pour la chaîne de réaffirmer sa double identité de chaîne de service public et de chaîne de télévision romande, dont le projet en matière de création télévisuelle pourrait être résumé de la manière suivante: mettre en scène des problématiques liées à la culture suisse avec les moyens de la téléréalité. Se positionnant en accentuant son identité de chaîne «respectable», la TSR, par la voix de la productrice de l'émission, insiste sur le caractère édifiant et l'intérêt scientifique du concept du programme:

L'enjeu est double: historique d'abord, puisqu'il s'agit de reconstruire le cadre et les conditions de vie de 1903. Sociologique ensuite, parce que cela donnera l'occasion à une famille de vivre une expérience proche de la nature ${ }^{16}$.

Le lieu de vie choisi pour mener cette "expérience» est présenté comme emblématique du patrimoine romand, dont l'émission doit permettre de transmettre une connaissance de façon ludique, en centrant les activités des participants sur la reproduction de gestes considérés comme représentatifs de cette époque et de cette région. Ainsi, placer une

15. Le "concept» du Mayen 1903 consiste à isoler les membres d'une famille dans un mayen d'alpage, situé dans le val d'Anniviers (canton du Valais), afin de les soumettre aux conditions de vie en vigueur en 1903, en leur faisant exécuter un certain nombre de tâches emblématiques du mode de vie de cette époque.

I6. Béatrice Barton citée dans Ch. Meichtry, «Ils vont vivre comme en 1903 », p. 3. 
famille dans un cadre qui simule les "conditions» de vie des Anniviards de l'époque de référence, renforcé par l'injonction qui leur est faite de reproduire certaines activités identifiées comme typiques, doit conduire les participants à adopter un mode de vie le plus proche possible de ce qui prévalait à l'époque ${ }^{17}$. Les discours qui concernent le lancement de l'émission dans la presse associent systématiquement le mayen à un ensemble de clichés discursifs sur "l'enchantement du paysage alpin", "l'authenticité du cadre de vie», «le décor idyllique». Les Alpes et la situation géographique du mayen sont ainsi reconnues comme des signes prégnants de l'identité romande. Dans une perspective critique, le sociologue Gabriel Bender, dont les interventions ont été reprises dans plusieurs journaux, dénonce le recours à une idéologie au centre de laquelle figure l'assimilation du paysage alpin à des valeurs supposées typiquement helvétiques:

Mayen 2003 [sic] est bien à l'image de la Suisse. Enfermée dans ses montagnes, isolée, absente de la compétition, petite chose harmonieuse, familiale, où le seul enjeu est de savoir comment répéter les gestes oubliés, accepter les corsets: travail, famille, patrie [...] dans les Alpes préservées du tourisme et de la technique ${ }^{18}$.

La position ici exprimée est également manifeste dans certains courriers de lecteurs qui dénoncent le contenu idéologique implicite du projet, en particulier quant au choix du cadre de vie et de sa représentation esthétisée. Cette approche critique est cependant minoritaire en regard de l'ensemble des articles consacrés au programme de la TSR, qui témoignent principalement d'une réception louangeuse quant à la démarche de la chaine et d'une satisfaction ressentie face à un spectacle rendant compte de la «beauté» du paysage représenté.

Toutefois, si l'unité culturelle romande se joue ainsi dans un rapport privilégié au paysage alpin comme renvoyant à un patrimoine culturel commun, certains discours participent à distinguer, au sein de celle-ci, des particularités qui renvoient aux origines cantonales des candidats.

17. «La famille Cerf évolue dans un décor, avec les objets et les conditions de 1903, elle accomplit les mêmes gestes, les mêmes tâches que les Anniviards de 1903 [...] mais elle le fait avec ses connaissances et sa culture de 2003" (Béatrice Barton, citée dans B. Barton, Le Mayen 1903, p. 7).

I8. Gabriel Bender, " "Le Mayen 1903” ou l'avenir à reculons», p. 18. 
Unicité et diversité de la culture romande: le critère de l'appartenance cantonale

La famille qui participe au programme, les Cerf, originaires de Courtemaîche dans le canton du Jura, est ainsi toujours présentée en mentionnant son origine jurassienne. Le choix de la TSR de favoriser une candidature non valaisanne, alors que le programme prévoit de documenter le mode de vie valant dans cette région au début du siècle, donne lieu à des prises de position de lecteurs et de spectateurs qui déplorent le fait que la TSR n'ait pas choisi une famille originaire du Valais pour participer à l'émission, ce qui, de l'avis de certains d'entre eux, aurait été plus authentique ${ }^{19}$. Dans le même sens d'une mise en avant du critère de l'appartenance régionale sur le mode de l'identité, la plupart des articles consacrés à l'émission intègrent le témoignage d'Anniviards, invités à réagir, en tant qu'habitants de la région représentée, sur le comportement de la famille et son adéquation avec le mode de vie valant à l'époque de référence mais aussi sur leur propre relation avec ce passé mythifié. La relation identitaire s'affirme ainsi sur le modèle du même: seuls les gens issus de cette culture localisée seraient à même de produire des comportements authentiques.

Le critère de l'identité entre les spectateurs et les participants s'exprime aussi dans les différences de réception entre les cantons, tant au niveau du traitement du programme dans la presse que pour ce qui concerne les taux d'audience. Ainsi, Le Nouvelliste, quotidien valaisan, consacre un article par semaine au programme de la TSR, en commentant les activités effectuées par les Cerf à partir de deux types de sources: les témoignages d'habitants de la région et le matériel d'archive de la presse régionale de l'époque. L'Impartial, quotidien distribué dans la région jurassienne, favorise quant à lui les témoignages de proches de la famille, et commente également leur retour dans leur village. En termes d'audience, le facteur d'identification semble également avoir un fort impact: les taux

19. La lettre d'une lectrice précise: «Je ne cautionne pas tout à fait Le Mayen 1903, car je pense qu'il aurait fallu prendre une famille valaisanne." (L. Glé, "Courrier des lecteurs», p. 18). Dans le même sens, un lecteur relève: "C'est sûr que si ça avait été des Valaisans qui avaient participé à cette expérience, on aurait été plus près des conditions d'époque. Mais je trouve que cette famille de Jurassiens se débrouille bien, et son accent, même s'il choque un peu dans la vallée, est très plaisant à l'oreille. " (Pascal Rion, cité dans V. Fragnière, «Il en reste 68’000!», p. 29). 
d'audience les plus élevés sont mesurés en Valais et la différence entre les cantons ruraux et les cantons citadins est également manifeste ${ }^{20}$.

Les programmes de téléréalité produits par la TSR à la suite du Mayen 1903 s'inscrivent dans la continuité de celui-ci, selon une logique de déclinaison aussi bien quant aux similitudes de concepts, exprimés dans une perspective sociale et pédagogique proche de celle qui était revendiquée dans le cas de Mayen 1903, qu'en regard du critère de l'appartenance cantonale des candidats. Ainsi, chaque programme est conçu pour mettre en scène des candidats issus de chacun des cantons romands. Super Seniors, dont l'argument d'utilité publique repose sur le fait qu'il doit permettre d'offrir une image de la retraite active et discuter du statut des retraités dans la société suisse, met en scène des participants issus de tous les cantons romands ${ }^{21}$. L'origine cantonale des participants structure le déroulement du tournage mais aussi le contenu diffusé puisque celui-ci est construit sous la forme d'épisodes, diffusés à un rythme hebdomadaire, qui relatent la visite dominicale qu'effectue le groupe des participants dans chacun des cantons romands.

Dîner à la ferme, qui repose sur une compétition entre agriculteurs invités à concocter le meilleur repas dont l'évaluation permettra de départager le gagnant, se structure sur une partition similaire. Chaque épisode est centré sur un des participants qui accueille les autres dans son canton. Les repas que composent les participants sont également conçus pour donner à goûter les spécialités locales, célébrant le «terroir» dont elles manifestent les particularités. Le cadre de vie, autant pour ce qui renvoie à l'activité agricole que pour son ancrage dans certaines spécificités paysagères, est mis en scène en détaillant ces caractéristiques. Sans prétendre à une analyse exhaustive des procédés de réalisation mis

20. "Le Mayen 1903 a réalisé un taux d'audience inespéré pour la TSR en fidélisant une moyenne de 257’000 spectateurs. Le Jura a été le plus assidu (66.1 pdm), suivi de Fribourg (62.8) et du Valais (59.4), des cantons où la tradition agricole est encore présente. A contrario, Genève arrive en queue avec 37.3\% de parts de marché.» (M.-C. Martin, "Le Mayen 1903” se termine sous la neige», p. 35).

2I. Dans la plupart des sources consacrées au lancement du programme, l'appartenance cantonale des participants est spécifiée, au risque de les constituer comme des stéréotypes (ainsi que l'implique l'utilisation de l'article défini): "Cet automne, les téléspectateurs suivront donc les pérégrinations de Bernadette, la vigneronne valaisanne, Cosette, l'enseignante neuchâteloise, Geneviève, la grand-maman venue du Jura, Irène, la couturière fribourgeoise, Jean-Louis, l'animateur radio de Genève, et tous leurs nouveaux amis.» (P. Monay, "Treize joyeux retraités prêts à s'éclater», p. 31). 
à l'œuvre dans ces programmes, nous en mentionnons un aspect récurrent qui repose sur une spatialisation, non plus par le biais des discours, mais par le biais du recours à certains codes iconographiques utilisés pour signifier le rapport des lieux dans lesquels vivent ou évoluent les participants et le paysage dans lequel ceux-ci sont inscrits.

Au même titre que l'appartenance cantonale et que le projet pédagogique, la représentation du paysage fonctionne en effet comme un des dénominateurs communs à ces programmes. Nous avons mentionné, dans le cas de Mayen 1903, les discours qui thématisent le rapport du patrimoine romand avec la représentation du paysage alpin. Dans Super Seniors, la situation des participants, logés dans un hôtel d'altitude, est l'occasion d'inscrire dans l'économie des épisodes des plans centrés sur le panorama alpin, mis en scène comme objet de contemplation ou encore comme objet spectaculaire. Dans le même sens, le dernier programme produit par la TSR est particulièrement révélateur de cette inscription des lieux d'habitation, valant comme exemples typiques de la vie villageoise contemporaine, en regard du paysage qui les entoure. Ainsi, chaque émission de Mon village a $d u$ talent débute par une vue aérienne de l'environnement paysager dans lequel est situé le village, pour se resserrer progressivement sur celui-ci. Le recours à la vue aérienne et le déplacement de la caméra opérée dans ces cas illustrent deux fonctions attribuées traditionnellement à la représentation du paysage: cadre de vie, territoire de l'essence culturelle, il est aussi construit comme objet d'attraction spectaculaire ${ }^{22}$. L'espace représenté est ainsi assimilé au cadre dans lequel se construit l'appartenance identitaire, les spécificités de celui-ci s'exprimant en retour par des particularités culturelles localisées. Yvan Droz discute de cette imbrication entre valeurs culturelles et paysage autour de la notion de "paysage naturalisé». Il montre comment s'opère le déplacement qui permet de constituer un donné objectif (l'environnement visuel perçu) en signe porteur de caractéristiques culturelles partagées par les membres de la communauté:

22. Réactivant ainsi certaines pratiques du panorama en vigueur dès le XVIII ${ }^{\mathrm{e}}$ siècle, cette représentation du paysage repose sur une réduction de l'identité suisse au paysage alpin, valant comme signe caractéristique de cette identité culturelle. A titre d'élargissement de cette problématique, on peut ainsi remonter à la conception du village suisse lors de l'exposition nationale de 1896, qui avait comme particularité de comprendre une montagne, élément que ces concepteurs jugeaient indispensable dans la représentation d'une Suisse ainsi réduite à ses composantes identificatoires (voir B. Crettaz, J. Michaelis-Germanier, Une Suisse miniature ou les grandeurs de la petitesse). 
Le paysage est un construit social qui naît de la rencontre entre un regard imprégné de valeurs et un espace présentant différentes caractéristiques. [...] Le paysage naturalisé n'est autre que l'illusion d'un paysage donné objectivement, d'un paysage qui va de soi. [...] Ainsi, les différences objectives sont associées ou confondues avec des valeurs subjectives, arbitraires ou culturelles qui sont alors perçues et conçues comme objectives ${ }^{23}$.

Les recours à la représentation paysagère, au sein des programmes de notre corpus, s'inscrivent parfaitement dans cette logique, où le paysage, considéré dans sa valeur de signe, fonctionne comme une ressource commune à ceux qui partagent l'appartenance à la même communauté culturelle.

Que cela se manifeste dans les discours qui accompagnent le lancement des émissions dans la presse, ou dans leur réalisation même, l'identité culturelle et son inscription dans un espace singularisé sont des aspects centraux de la réception de la téléréalité en Suisse romande et de la programmation de la TSR en matière de téléréalité. Le choix d'une thématique transversale comme celle de la notion d'espace pour explorer notre corpus permet de dégager le rôle central des opérations d'inscription des programmes de téléréalité au sein d'un espace culturel existant préalablement à leur diffusion en Suisse romande, mais également les procédés mis en œuvre pour construire cet espace culturel comme un lieu de détermination identitaire pour les spectateurs, journalistes et producteurs de contenus ancrés dans le territoire romand. Ainsi, que cela concerne les discours de presse ou les modalités d'écriture des programmes étudiés, la téléréalité fonctionne comme un support pour construire des signes de l'appartenance à la culture romande. Sans épuiser toutefois les analyses qui pourront être menées sur ce corpus, cette contribution aura permis d'étudier les implications spatiales de la représentation et de l'articulation discursive de l'identité culturelle dans le cas de la réception de la téléréalité en Suisse romande.

Charlotte Bouchez

Université de Lausanne

23. Y. Droz, Pour une anthropologie politique du paysage, p. 11-15. 


\section{BIBLIOGRAPHIE}

\section{Sources}

(ats), «La télé-réalité débarque à la TSR», L'Impartial, 24 octobre 2003, p. 25.

Bender, Gabriel, " "Le Mayen 1903” ou l'avenir à reculons", Le Temps, 14 octobre 2003, p. 18.

Elgass, Jean, «Le succès de Loft Story déborde en Suisse», 24 heures, 8 mai 2001, p. 33.

Fragnière, Vincent, "Il en reste 68'000!», Le Nouvelliste, 18 octobre 2003, p. 29.

GlÉ, Liliane, "Courrier des lecteurs. Vie paysanne», TV Guide, 15 novembre 2003, p. 18.

Iмноғ, Michel, "Non à la télé "trou de serrure" et aux "émissions poubelles" ", 24 heures, 25 avril 2001, p. 39.

KRÄHEnbüHL, Jean-François, «Béatrice Barton, reine de l'information divertissement", Le Matin, 20 juin 2003, p. 4.

Martin, Marie-Claude, " "Le Mayen 1903 ” se termine sous la neige», Le Temps, 21 novembre 2003, p. 35.

Meichtry, Chiara, "Ils vont vivre comme en 1903», Le Matin, 12 juillet 2003, p. 3.

Monay, Patrick, Bressoud, Estelle, "Treize joyeux retraités prêts à s'éclater", 24 heures, 10 mai 2005, p. 31.

Nicoud, Elisabeth, «La TSR résiste à la tempête», 24 heures, 22 janvier 2002, p. 34.

Richon, Nadine, "Le nouveau directeur de la TSR opère des changements avec un gant de velours", Le Temps, 2 mars 2001, p. 42.

Schouwey, Pascal, «Mon œil», Tribune de Genève, 26 avril 2001, p. 4. 


\section{Travaux}

Appadurai, Arjun, Après le colonialisme. Les conséquences culturelles de la globalisation, trad. de l'anglais par Françoise Bouillot, Paris, Payot, 2005 (1996).

Barker, Chris, Television, Globalization and Cultural Identities, London, Open University Press, 2003 (1999).

Barton, Béatrice, Le Mayen 1903, Lausanne/Genève, TSR/Favre, 2003.

Cola, Marta, "Media Studies and Social Geography: a Combination of Theories in an Empirical Research ", Studies in Communication Sciences, 6/2 (2006), p. 269-280.

Crettaz, Bernard, Michaelis-Germanier, Juliette, Une Suisse miniature ou les grandeurs de la petitesse, Genève, Musée d'ethnographie de la Ville de Genève, 1982.

Cucco, Marco, "The Borders of the Domestic Market and Their Importance for the Economy of the Film Industry: The Swiss Case Study", European Journal of Communication, 25/2 (2010), p. 153-167.

Droz, Yvan (éd.), Pour une anthropologie politique du paysage. Valeurs et postures paysagères des montagnes suisses, Paris, Editions Karthala, 2009.

Kredens, Elodie, "La télé-réalité entre adaptabilité des formats et spécificités nationales: le cas de l'émission Big Brother", in Colloque international "Mutations des industries de la culture, de l'information et de la communication", Paris, 2006, p. 10-11.

Morley, David, Robins, Kevin, Spaces of Identity: Global Media, Electronic Landscape and Cultural Boundaries, London, Routledge, 1995. 
